\title{
Power Efficiency Calculation of Acoustic Energy Transmission by a Stepped-Plate Radiator
}

\author{
Yonghwan Hwang, Wonkyu Moon* \\ Department of Mechanical Engineering, Pohang University of Science and Technology, Pohang, Korea \\ Email: *wkmoon@postech.ac.kr
}

How to cite this paper: Hwang, Y. and Moon, W. (2017) Power Efficiency Calculation of Acoustic Energy Transmission by a Stepped-Plate Radiator. Energy and Power Engineering, 9, 232-240. https://doi.org/10.4236/epe.2017.94016

Received: March 25, 2017

Accepted: April 23, 2017

Published: April 26, 2017

Copyright $\odot 2017$ by authors and Scientific Research Publishing Inc. This work is licensed under the Creative Commons Attribution International License (CC BY 4.0).

http://creativecommons.org/licenses/by/4.0/ (c) (i) Open Access

\begin{abstract}
One performance measure of in-air ultrasonic radiators, such as wireless power transmission, is the power efficiency of the transducers. The efficiency of most in-air acoustic radiators is low, even at ultrasonic frequencies; however, a large radiating plate with steps introduced by Gallego-Juarez et al., can provide efficient radiation. Their in-air acoustic radiator consists of a Langevin transducer for wave excitation, a mechanical amplifier, and a stepped plate with a large radiating area. This study describes a design processing technique for a stepped-plate radiator developed for optimum energy transmission at the target point in air. The total efficiency required to transfer the acoustic energy was divided into three categories, and the design parameters of each category were calculated to maximize the efficiency. This design technique allows optimum acoustic radiation efficiency and maximum acoustic energy transmission depending on various acoustic energy transfer conditions.
\end{abstract}

\section{Keywords}

Stepped-Plate Radiator, Maximum Radiation Efficiency, Acoustic Energy Transmission

\section{Introduction}

A number of wireless energy transmission technologies, including inductive coupling, capacitive coupling, magnetic coupling, microwaves, light waves, and acoustic radiation, are currently proposed, under investigation, or applied. One type, acoustic wireless transmission, uses sound propagation energy in the medium and can reduce the effect of electromagnetic waves. However, in-air acoustic radiation has a low radiation efficiency because of the large impedance mismatch between the air and the acoustic generator [1] [2]. Therefore, one of the most important performance measures of in-air acoustic radiators is their power efficiency, which can be improved by addressing this mismatch. 
The energy transfer system for acoustic energy differs depending on the transmission target. Maximization of the efficiency of wireless acoustic energy transmission at a particular point requires a transducer with high acoustic radiation efficiency and depends on the directivity of the acoustic beam. The use of a small region for the energy receiving position will necessitate a high directional sound beam or a focused sound beam. In other words, efficient energy transmission is impossible with a nondirectional sound beam, such as an omnidirectional sound generator, even with a high-efficiency of the acoustic generator is high.

The stepped plate radiator has been adopted to provide high directional, high acoustic radiation efficiency and a high intensity sound beam [3] [4] [5] [6]. The Gallego-Juarez group introduced a stepped-plate radiator that increases the acoustic radiating area of the projector without changing the size of its driver [3] [4] [5] [6]. However, increases in the size of the radiating plate cause flexural vibration of the plate and lead to discontinuous plate phases and undesirable directivity patterns. The Gallego-Juarez group improved the directivity pattern through the use of steps with heights equal to half the wavelength of sound in air. The basic idea underlying this concept was to compensate for the discrete phase difference by introducing steps in the vibrating plate. This type of transducer offers a large radiation area and good directivity at high frequency. It also has a simple structure and can reach a high radiation efficiency while producing high-intensity acoustic waves [3] [4] [5] [6]. This transducer can be used for acoustic energy transmission in air.

Many parameters in the design of a stepped-plate radiator for in-air energy transmission may affect the acoustic radiation efficiency. The design of a stepped-plate radiator optimized for acoustic energy transmission must take into consideration the optimum design of each part, as well as the integrated system and boundary conditions.

In the present study, we introduce a method for designing a stepped-plate radiator for wireless acoustic energy transmission. First, we suggest the driving frequency and the radiating area at a target receiving point. This frequency and radiating area are then used to calculate the detailed dimensions of the steppedplate radiator for maximum acoustic radiation efficiency. Using these results, we can then identify the final acoustic wireless energy transmission efficiency of the stepped plate radiator.

\section{Concept of Acoustic Wireless Energy Transmission}

Figure 1 shows the schematic view of the acoustic wireless energy transmission system. Acoustic energy transmission has three efficiencies: transmitter efficiency, propagation efficiency, and the receiver efficiency. The transmitter efficiency is related to the structure of the radiator, the material, and the radiation impedance of the plate. The propagation efficiency is related to the directivity and the frequency of the sound beam. The receiver efficiency is related to the structure and the electrical energy storage system of the receiver.

Our design example has three fixed simulation conditions: 


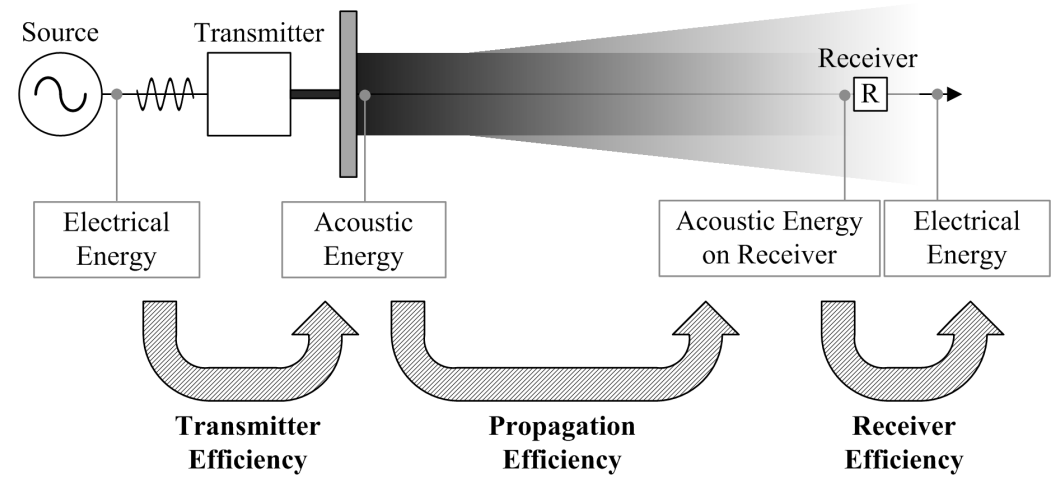

Figure 1. The schematic view of the acoustic wireless energy transmission system.

- The shape of the receiver is a point.

- The distance between the acoustic radiator and the receiver is $3 \mathrm{~m}$.

- The receiver efficiency at a given position is $100 \%$.

These fixed simulation conditions can be changed according to the application field.

\section{Propagation Efficiency}

A parametric study is often performed to determine the appropriate values of various design parameters as quickly as possible. With such applications, the radiation power efficiency must be maximized to minimize the power loss. In addition, the directional pattern of the radiated acoustic sounds is important because power loss during transmission can be minimized if most of the acoustic power emitted by the acoustic radiator is transmitted to the receiver. In other words, a large sharp major lobe is required in the acoustic radiation pattern.

First, we should check whether the efficiency of the radiated acoustic wave can be maximized at a receiver distance of $3 \mathrm{~m}$ from the radiating surface of the stepped-plate radiator. This can be achieved as the driving frequency and radius of the plate.

The driving frequency and the radiation area of the plate can be determined by considering the acoustic wave emitted by the radiator. When an acoustic wave propagates in air, spherical spreading and attenuation should be considered [7]. Spherical spreading is an acoustic phenomenon whereby the energy of the acoustic wave is inversely proportional to the square of the distance. The location on the surface where the spherical spreading begins can be calculated from the Rayleigh distance, $R_{d}=\pi a^{2} / \lambda$, when the radiation area is a rigid circular plate. Attenuation occurs due to losses in the medium and, in air, is proportional to the square of the frequency. Spherical spreading increases at low frequencies because of the short Rayleigh distance. At high frequencies, attenuation may increase. Therefore, an optimum frequency for a given transmission distance is expected due to the opposite trends in the effects of spherical spreading and attenuation. The receiver efficiency can be calculated as follows [7]:

$$
\eta_{r e}=\frac{I_{r e}}{\frac{1}{2} \operatorname{Re}\left[Z_{r}\right] U_{\text {plate }}^{2}}
$$


where $Z_{r}$ and $U_{\text {plate }}$ are the acoustic radiation impedance and velocity of the rigid circular plate, respectively [7]. The received acoustic intensity at a given location is $I_{r e}=|p|^{2} / 2 \rho_{0} c \quad$ [7].

Figure 2 shows simulation results for the relationship between the radius of the plate and frequency. The frequency and the radius of the plate should be selected based on the specific application. In this study, we determined the optimum frequency to be $70 \mathrm{kHz}$ and the optimum radius to be $0.1 \mathrm{~m}$, to obtain the maximum received acoustic efficiency at $3 \mathrm{~m}$.

\section{Transmitter Efficiency}

The design parameters are related to the configuration of the stepped-plate radiator. Figure 3 shows the installation conditions of the stepped-plate radiator used for the simulation. The Langevin transducer consists of two PZT rings with head and tail components, which are combined using an elongated bolt that pre-stresses the contact interfaces between the PZT rings and the head or tail component (see Figure 3).

In Section 3, the resonance frequency and the radius of the plate were determined. Thus, the overall design process for a stepped-plate radiator can be summarized as follows:

1) Design the driving components, including the Langevin transducer and stepped horn. The resonance mode shape of the driving components is the second longitudinal mode; this is because the node points are located between the two PZT ring elements and between the different cross-sectional areas of the stepped horn (Line 1 in Figure 3).

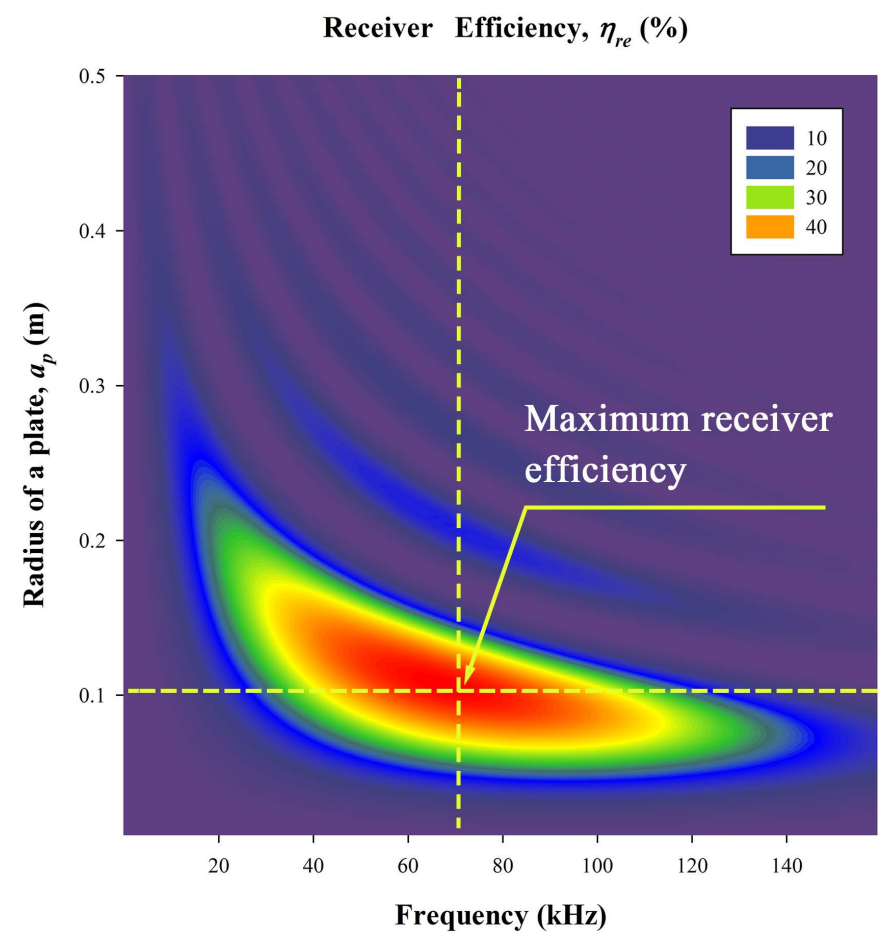

Figure 2. Receiver efficiency relationship between the radius of the plate and frequency. 


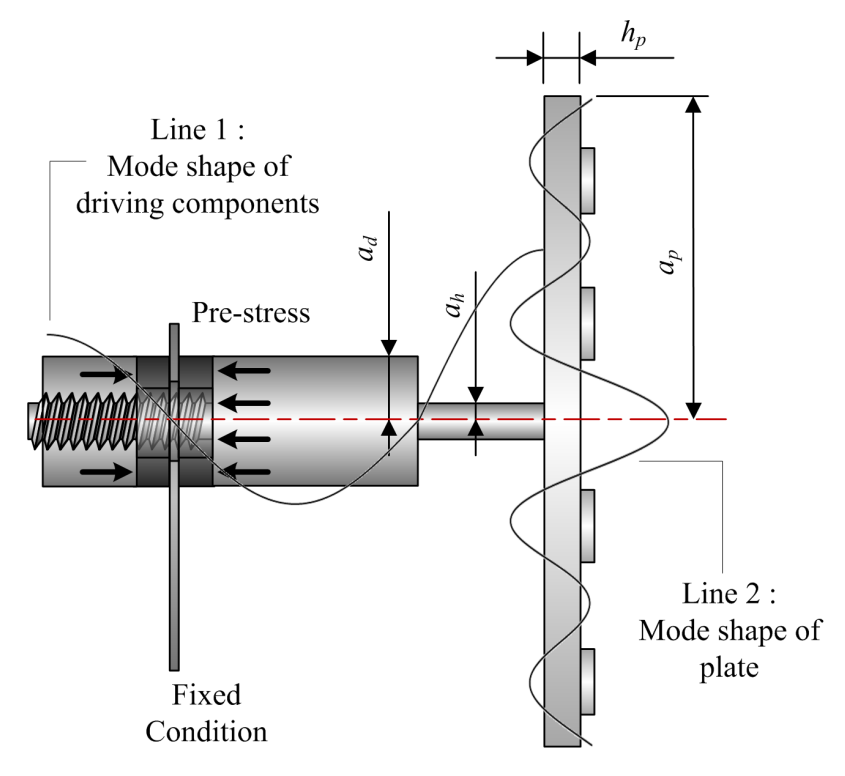

Figure 3. Installation conditions of the stepped-plate radiator.

2) Design a plate with identical resonance frequency to that of the driving components, and attach it to the end of the stepped horn.

3) Calculate the acoustic radiation efficiency of the designed stepped-plate radiator.

In this research, the simulations were calculated by the commercial FEM package (COMSOL Multiphysics) and the material properties of each part are in Table 1. Figure 4 shows the boundary conditions and simulation conditions of the stepped-plate radiator in COMSOL Multiphysics. We use the $2 \mathrm{~d}$ axisymmetric model since the shape of a stepped-plate radiator. The interface $\mathrm{A}$ is the far-field calculation condition which means the perfect matched layer and no reflection of the acoustic waves.

The transmitter efficiency of the stepped-plate radiator $\left(\eta_{t r}\right)$ can be defined as the ratio of the radiated acoustic power to the electrical power input: i.e.,

$$
\eta_{t r}=\frac{P_{\text {out }}}{P_{\text {in }}}=\frac{\int_{\text {interface } A} I_{t r} d S}{\operatorname{Re}[V] \operatorname{Re}[i]}
$$

where $V$ is the voltage applied to the PZT ceramics, $i$ is the consumed current of the PZT ceramics, and $I_{t r}$ is the acoustic intensity at the interface A. The design parameters for the stepped-plate radiator are the length of the driving components $L_{\text {drive }}$ the radius of the plate $a_{p}$, the thickness of the plate $h_{p}$, the radius of the horn $a_{h}$, and the radius of the driver $a_{d \cdot}$. The length of the driving components is directly related to the resonance frequency [8]. The resonance frequency is not included in the design parameters because it is given by the operating frequency, which is determined via a calculation of the propagation efficiency, as described in Section 3. Using the four variables $a_{p}, h_{p}, a_{h}$ and $a_{d}$ we can define the following three dimensionless parameters to investigate the performance of the radiator. 


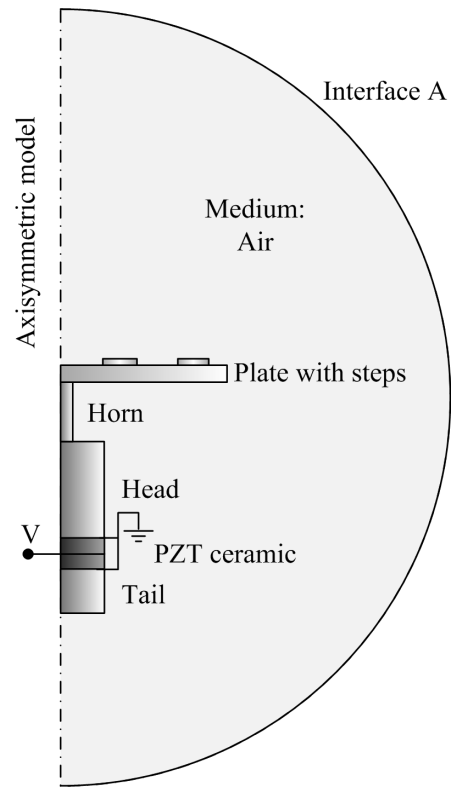

Figure 4. Boundary conditions of the stepped-plate radiator.

Table 1. Material properties of the aluminum and PZT ceramic.

\begin{tabular}{|c|c|c|c|}
\hline Materials & Properties & & Values \\
\hline \multirow{4}{*}{$\begin{array}{l}\text { Aluminum } \\
(\mathrm{Al}-7075)\end{array}$} & Elastic modulus & $E$ & $72 \mathrm{GPa}$ \\
\hline & Mass density & $\rho$ & $2780 \mathrm{~kg} / \mathrm{m}^{3}$ \\
\hline & Poisson's ratio & $v$ & 0.33 \\
\hline & Mechanical quality factor & $Q_{m}$ & 2000 \\
\hline \multirow{13}{*}{$\begin{array}{c}\text { PZT ceramic } \\
\text { (C-21 by } \\
\text { Fuji ceramics })\end{array}$} & & $s_{11}^{E}$ & $12.0 \times 10^{-12} \mathrm{~m}^{2} / \mathrm{N}$ \\
\hline & & $s_{12}^{E}$ & $-3.4 \times 10^{-12} \mathrm{~m}^{2} / \mathrm{N}$ \\
\hline & Elastic modulus & $s_{13}^{E}$ & $-5.5 \times 10^{-12} \mathrm{~m}^{2} / \mathrm{N}$ \\
\hline & & $S_{33}^{E}$ & $14.7 \times 10^{-12} \mathrm{~m}^{2} / \mathrm{N}$ \\
\hline & & $S_{44}^{E}$ & $41.7 \times 10^{-12} \mathrm{~m}^{2} / \mathrm{N}$ \\
\hline & \multirow{3}{*}{ Piezoelectric constants } & $d_{31}$ & $-117 \times 10^{-12} \mathrm{~m} / \mathrm{V}$ \\
\hline & & $d_{33}$ & $232 \times 10^{-12} \mathrm{~m} / \mathrm{V}$ \\
\hline & & $d_{15}$ & $582 \times 10^{-12} \mathrm{~m} / \mathrm{V}$ \\
\hline & \multirow{2}{*}{ Relative dielectric constants } & $s_{11}^{T} / \varepsilon_{0}$ & 1854 \\
\hline & & $s_{33}^{T} / \varepsilon_{0}$ & 1184 \\
\hline & Mechanical quality factor & $Q_{m}$ & 1235 \\
\hline & Mass density & $\rho$ & $7600 \mathrm{~kg} / \mathrm{m}^{3}$ \\
\hline & Dissapation factor & $\operatorname{Tan} \delta$ & $0.3 \%$ \\
\hline
\end{tabular}

$$
\alpha=\frac{a_{h}}{a_{d}}, \beta=\frac{a_{h}}{a_{p}}, \gamma=\frac{h_{p}}{a_{p}}
$$

We suggest the dimensionless parameters; $\alpha, \beta$ and $\gamma$, where $\alpha$ is the amplification ratio of the stepped horn, $\beta$ is the ratio of the cross-section of the stepped 
horn to that of the plate, and $\gamma$ is the ratio of the radius to the thickness of the plate.

A stepped-plate radiator has steps to compensate for the discrete phase difference in the vibrating plate. The height of the steps should be carefully selected, since the fabrication limit due to the extremely thin circular plate. The minimum thickness of the plate in this case may be $5 \mathrm{~mm}$. Thus, as shown in Figure 5(a),

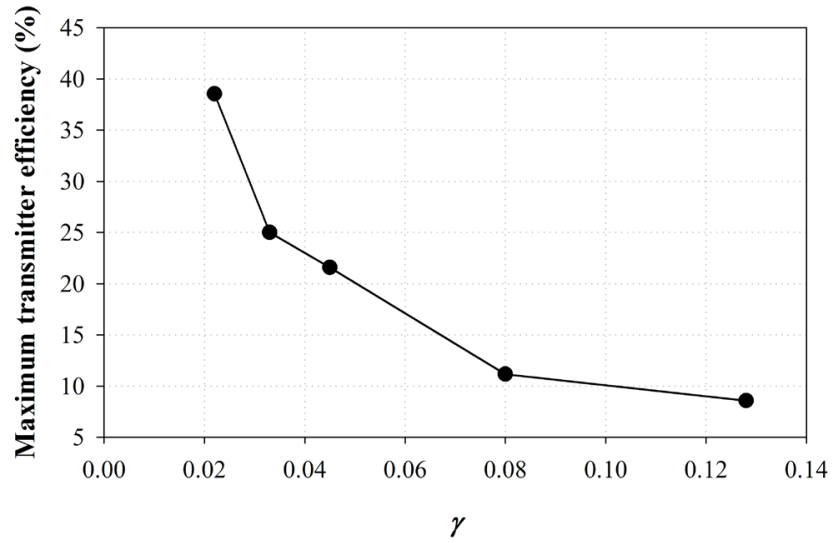

(a)

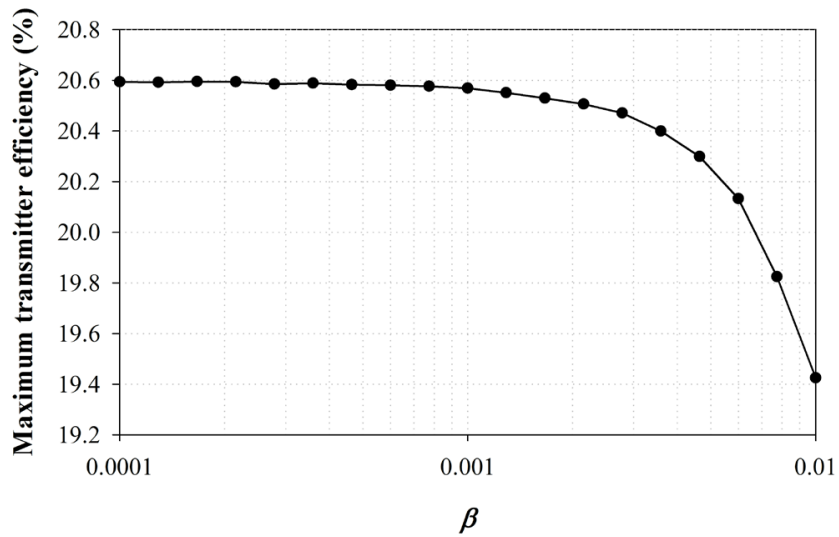

(b)

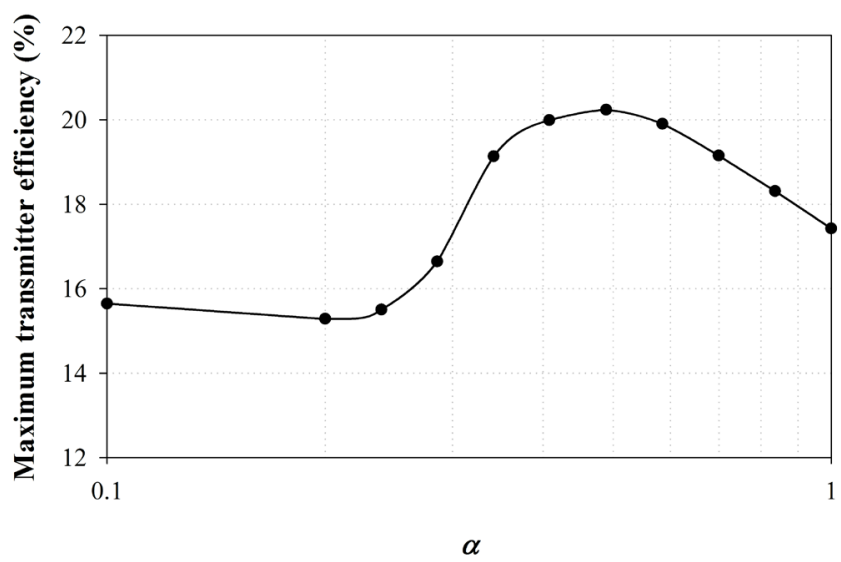

(c)

Figure 5. The maximum transmitter efficiency of the stepped-plate radiator (a) which is related to the $\gamma$, (b) $\beta$, and (c) $\alpha$. 
for the $8^{\text {th }}$ mode, we have a minimum of $\gamma=0.045$ when the radius of the plate is $0.1 \mathrm{~m}$ and the driving frequency is $70 \mathrm{kHz}$. We find $2 \mathrm{ka}=256.4$ for the $8^{\text {th }}$ mode.

Optimum values of $\alpha, \beta$ and $\gamma$ should be calculated via a parametric study to maximize the acoustic radiation efficiency of a stepped-plate radiator. The dimensionless parameters $\beta$ is used to calculate the maximum transmitter efficiency when $\gamma$ depends on the selected mode number. Figure 5(b) shows the transmitter efficiency, $\eta_{t r}$, for the $8^{\text {th }}$ mode of the plate. We find $\gamma=0.045$, and the optimum value of $\beta$ is 0.000167 , and the maximum transmitter efficiency is $20.6 \%$. It follows that $\beta$ is optimal to maximize the transmitter efficiency.

Using Equation (3), we find an optimal value of $\beta$ is 0.000167 , and the radius of the horn is ah $=0.0167 \mathrm{~mm}$, which is too small to fabricate the stepped-plate radiator, as shown in Figure 3 . In the parametric calculation, we restrict the minimum radius of the horn to $2 \mathrm{~mm}$, and $\beta=0.02$. Figure 5 (c) shows the relationship between the transmitter efficiency and $\alpha$, when $\beta=0.02$ and $\gamma=0.045$. The maximum transmitter efficiency is $20.2 \%$, which differs from that shown in Figure 5 (b) by $0.4 \%$. The optimum value of $\alpha$ is 0.489 , and the optimum radius of the driver is $a_{d}=4 \mathrm{~mm}$.

Based on the simulation results, the dimensionless parameters $\alpha$ and $\beta$ are determined to achieve the maximum power efficiency. The value of $\gamma$ is restricted by the fabrication limit of a plate. Thus, by considering the simulation results and other constraints for the stepped-plate radiator, we determine the design parameters that yield the maximum power efficiency for a stepped-plate acoustic radiator as follows. For an operating frequency of $70 \mathrm{kHz}$, we have $a=100 \mathrm{~mm}$, $t_{p}=4.5 \mathrm{~mm}, a_{h}=2 \mathrm{~mm}$ and $a_{d}=4 \mathrm{~mm}$. The power efficiency of the resulting stepped-plate radiator is $20.2 \%$.

\section{Conclusions}

The in-air acoustic wireless energy transmission with a stepped-plate radiator described here has high efficiency, high directivity, and a high-intensity acoustic beam. We classify three types of acoustic efficiency: transfer, propagation, and the receiver efficiency. The receiver efficiency was assumed to have a maximum value and the shape of the receiver is a point. For the optimum propagation efficiency at a $3 \mathrm{~m}$ distance, the driving frequency and the radius of the plate are 70 $\mathrm{kHz}$ and $0.1 \mathrm{~m}$, respectively. The design parameters of the stepped-plate radiator can be represented as 4 variables: the radius of the plate $a_{p}$, the thickness of the plate $h_{p}$, the radius of the horn $a_{h}$, and the radius of the driver $a_{d}$. Using these variables, we can obtain the 3 dimensionless parameters; $\alpha, \beta$, and $\gamma$, and optimum simulation results of $\alpha=0.489, \beta=0.02$, and $\gamma=0.045$, taking into consideration the boundary and installation condition of the stepped-plate radiator. The final maximum transmitter efficiency is $20.2 \%$.

This design technique achieves an optimum acoustic radiation efficiency of the stepped-plate radiator, as well as a maximum acoustic energy transmission, for various acoustic energy transfer conditions in the air. 


\section{Acknowledgements}

This work was supported by the National Research Foundation of Korea (NRF) grant funded by the Korea government (MSIP) (NO. 2016R1E1A2A02945515).

\section{References}

[1] Lee, H., Kang, D. and Moon, M. (2009) A Micro-Machined Source Transducer for a Parametric Array in Air. The Journal of the Acoustical Society of America, 125, 1879-1893. http://dx.doi.org/10.1121/1.3081385

[2] Moon, M. and Lee, H. (2007) Ultrasonic Ranging System and Method Thereof in Air by Using Parametric Array. U.S. Patent, 7, 196-970.

[3] Barone, A. and Gallego-Juarez, J.A. (1971) Flexural Vibrating Free-Edge Plates with Stepped Thicknesses for Generating High Directional Ultrasonic Radiation. The Journal of the Acoustical Society of America, 51, 953-959. http://dx.doi.org/10.1121/1.1912944

[4] Gallego-Juarez, J.A. and Rodriguez-Corral, G. (1973) Piezoelectric Transducer for Air-Borne Ultrasound. Acta Acustica United with Acustica, 29, 234-239.

[5] Gallego-Juarez, J.A., Rodriguez-Corral, G. and Gaete-Garreton, L. (1978) An Ultrasonic Transducer for High Power Applications in Gases. Ultrasonics, 16, 267-271.

[6] Gallego-Juarez, J.A. (2010) High-Power Ultrasonic Processing: Recent Developments and Prospective Advances. Physics Procedia, 3, 35-47.

[7] Kinsler, L.E., Frey, A.R., Coppens, A.B. and Sanders, J.V. (2000) Fundamentals of Acoustics. 4th Edition, John Wiley \& Sons, Inc, New York.

[8] Sherman, C.H. and Butler, J.L. (2007) Transducers and Arrays for Underwater Sound. Springer, New York. https://doi.org/10.1007/978-0-387-33139-3

Submit or recommend next manuscript to SCIRP and we will provide best service for you:

Accepting pre-submission inquiries through Email, Facebook, LinkedIn, Twitter, etc. A wide selection of journals (inclusive of 9 subjects, more than 200 journals)

Providing 24-hour high-quality service

User-friendly online submission system

Fair and swift peer-review system

Efficient typesetting and proofreading procedure

Display of the result of downloads and visits, as well as the number of cited articles

Maximum dissemination of your research work

Submit your manuscript at: http://papersubmission.scirp.org/

Or contact epe@scirp.org 